\title{
Excited-State Intramolecular Proton Transfer in Five-Membered Hydrogen-Bonding Systems: 2-Pyridyl Pyrazoles
}

\author{
Wei-Shan Yu, ${ }^{\dagger}$ Chung-Chih Cheng, ${ }^{\dagger}$ Yi-Ming Cheng, ${ }^{\dagger}$ Pei-Chi Wu, ${ }^{\ddagger}{ }^{\text {Yi-Hwa Song }},{ }^{\ddagger}$ Yun Chi, ${ }^{*} \neq$ and \\ $\mathrm{Pi}-\mathrm{Tai} \mathrm{Chou}^{*}, \dagger$ \\ Department of Chemistry, National Taiwan University, 106, Taipei, Taiwan R.O.C., and Department of Chemistry, \\ National Tsing Hua University, 300, Hsinchu,Taiwan R.O.C.
}

Received March 31, 2003; E-mail: chop@ntu.edu.tw

Since the seminal studies on the excited-state intramolecular proton transfer (ESIPT) reaction in salicylic deivatives, ${ }^{1}$ numerous ESIPT molecules have been discovered and investigated to shed light on their corresponding spectroscopy and dynamics. ${ }^{2}$ Most ESIPT molecules possess six-membered ring types of a strong intramolecular hydrogen bond between $\mathrm{O}-\mathrm{H}($ or $\mathrm{N}-\mathrm{H})$ and $\mathrm{C}=\mathrm{O}$ (or pyridinic nitrogen) groups, in which the intramolecular proton transfer in the ${ }^{1} \pi \pi^{*}$ state $^{3}$ commonly reveals a negligible barrier in nonpolar solvents and may proceed during the period of lowfrequency vibrational motions associated with the hydrogen bond. ${ }^{4-7}$

From the fundamental viewpoint, searching for the ESIPT reaction with a finite, well-defined barrier is of great importance to gain detailed insights into the reaction potential energy surface. To achieve this goal, conjugated pyrrole-pyridine systems such as $1-H$-pyrrolo[3, 2- $h$ ]quinolines ${ }^{8}$ possessing an $\mathrm{N}-\mathrm{H} \cdots \mathrm{N}$ type of fivemembered hydrogen bond have received much attention. Under similar proton donor/acceptor strengths, the intramolecular hydrogenbonding (HB) strength is empirically on the order of $6>5 \gg$ fourmembered systems due to the steric and orientation effects. Unlike the $\mathrm{O}-\mathrm{H}$ site that has certain rotational degrees of freedom, the orientation effect of the pyrrolic $\mathrm{N}-\mathrm{H}$ site being restricted toward a specific direction becomes more critical for the HB formation. Furthermore, the photoacidity of the pyrrolic hydrogen is in general weaker than that of the phenolic hydrogen. As a result, ESIPT in five-membered HB pyrrole-pyridine systems may be associated with an appreciably large barrier. Unfortunately, to our knowledge, none of the five-membered $\mathrm{N}-\mathrm{H} \cdots \mathrm{H}$ HB systems have yet been reported to exhibit intrinsic ESIPT. Instead, similar to the fourmembered proton donor/acceptor systems such as the 7-azaindoles, ${ }^{9}$ this class of molecules is well-known to undergo a guest molecule (including the protic solvents)-catalyzed excited-state proton-transfer reaction. ${ }^{8}$

In this study, we have alternatively designed and synthesized the conjugated 2-pyridyl pyrazole systems where the $\mathrm{N}(1)-\mathrm{H}$ acidity is enhanced via the electron-withdrawing property of the proximal nitrogen atom $(\mathrm{N}(2)$, see Scheme 1) in the pyrazole ring. Scheme 1 shows the molecular drawings of 5-(2-pyridyl) $1-H$ pyrazoles $1 \mathbf{a}-\mathbf{d}$, possessing various substituents at the -3 position. ${ }^{10}$ Both X-ray structural analyses and molecular modeling indicate that the $\mathrm{N}(1)-\mathrm{H} \cdots \cdot \mathrm{N}\left(1^{\prime}\right) \mathrm{HB}$ isomer is the predominant species for $\mathbf{1 a}-\mathbf{d}$ in the ground state (see Supporting Information).

Prototypical absorption and emission spectra of $\mathbf{1 a}$ in thoroughly dry cyclohexane are depicted in Figure 1 . Upon $\mathrm{S}_{0} \rightarrow \mathrm{S}_{1}\left(\pi \pi^{*}\right)$ excitation $\left(\lambda_{\max } \approx 290 \mathrm{~nm}, \epsilon_{290} \approx 1.2 \times 10^{4} \mathrm{M}^{-1} \mathrm{~cm}^{-1}\right)$, dual fluorescence was observed maximized at $320 \mathrm{~nm}$ (the $\mathrm{F}_{1}$ band) and $585 \mathrm{~nm}$ (the $\mathrm{F}_{2}$ band). The excitation spectra monitored at $\mathrm{F}_{1}$ and

$\dagger$ Department of Chemistry, National Taiwan University, 106, Taipei, Taiwan Departme
R.O.C.

¥ Department of Chemistry, National Tsing Hua University, 300, Hsinchu,Taiwan R.O.C.

10800 - J. AM. CHEM. SOC. 2003, 125, 10800-10801

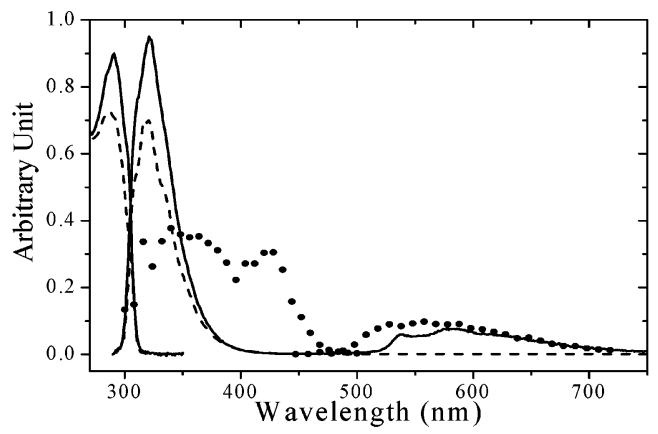

Figure 1. The absorption and emission spectra of (a) $1 \mathrm{a}(-), 1.0 \times 10^{-5}$ M), (b) 1a-1Me (- -) and (c) 1a-1'Me ( $\cdots)$ in cyclohexane. The excitation wavelength is $280 \mathrm{~nm}$.

Scheme 1. Structures and Proposed ESIPT Mechanism for the Studied Systems

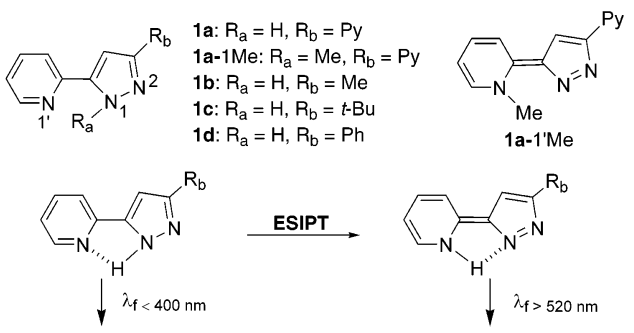

$F_{2}$ bands are identical, and are effectively the same as the $S_{0} \rightarrow S_{1}$ absorption spectral feature, indicating that both emission bands originate from the same ground-state precursor. In a comparative study, 1a-1Me (see Scheme 1), which serves as a nonproton-transfer model, exhibits a normal Stokes shifted emission maximum at $\sim 318$ $\mathrm{nm}$. Conversely, the proton-transfer tautomer analogue $1 \mathbf{1 a}-1^{\prime} \mathbf{M e}$ reveals a single fluorescence maximum at $\sim 570 \mathrm{~nm}$ of which the spectral feature is similar to that of the $\mathrm{F}_{2}$ band. We thus conclude that ESIPT takes place in 1a, resulting in an anomalously large Stokes-shifted $\left(>10000 \mathrm{~cm}^{-1}\right)$ proton-transfer tautomer emission. Similar dual emissions were resolved in $\mathbf{1 b}-\mathbf{d}$, of which the corresponding photophysical properties are listed in Table 1.

In contrast to the exclusive tautomer emission resulting from the ultrafast proton-transfer reaction for most ESIPT molecules, ${ }^{4}$ the appearance of dual emissions in $\mathbf{1} \mathbf{a}-\mathbf{d}$ in which the normal fluorescence is prevailing is remarkable. It implies the existence of an appreciably high barrier associated with ESIPT so that the decay dynamics of the locally excited $S_{1} \rightarrow S_{0}$ relaxation is competitive with the rate of proton transfer. Further insight into the reaction mechanism was gained from the dynamic studies. Single-exponential decay kinetics were resolved for the $\mathrm{F}_{1}$ band of $1 \mathbf{a}$, and a lifetime was fitted to be $\sim 85 \mathrm{ps}\left(\chi^{2}=1.02\right)$ at $298 \mathrm{~K}$, while the $\mathrm{F}_{2}$ band is apparently composed of rise and decay compo- 
Table 1. Photophysical Parameters of $\mathbf{1 a}-\mathbf{d}$ Measured in Cyclohexane at $298 \mathrm{~K}$

\begin{tabular}{cccccc}
\hline & $\lambda_{\max }^{\mathrm{F}_{1}}\left(\Phi^{\mathrm{a}}\right)^{a}$ & $\lambda_{\max }^{\mathrm{F}_{2}}(\Phi)^{a}$ & $\mathrm{~F}_{1}{ }^{b}$ & $\mathrm{~F}_{2}{ }^{b}$ & $\Delta E^{d}$ \\
\hline 1a & $320(19)$ & $585(1.5)$ & 85 & $80(-0.071)^{c}$ & 2.03 \\
& & & & $275(0.065)$ & \\
1b & $320(21)$ & $577(0.5)$ & 115 & $120(-0.017)$ & 2.22 \\
& & & & $242(0.021)$ & \\
1c & $320(25)$ & $577(0.2)$ & 125 & $130(-0.042)$ & 2.62 \\
& & & & $213(0.035)$ & \\
1d & $322(9)$ & $590(0.3)$ & 74 & $76(-0.070)$ & 1.76 \\
& & & & $175(0.061)$ & \\
\hline
\end{tabular}

${ }^{a} \Phi \times 10^{3} .{ }^{b}$ Picoseconds ${ }^{c}$ Numbers in the parenthesis are preexponential factors of the fitting eq $F(t)=a_{1} \mathrm{e}^{-k_{1} t}+a_{2} \mathrm{e}^{-k_{2} t}{ }^{d} \Delta E$ (in kcal/ mol) measured in methylcyclohexane. $\lambda$ in $\mathrm{nm}$.

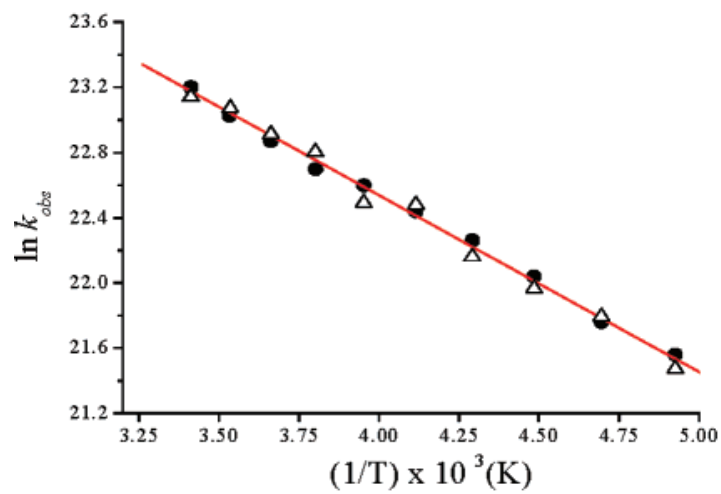

Figure 2. The plot for $\ln k_{\mathrm{obs}}$ versus the reciprocal of temperatures in methylcyclohexane: 1a- $h(\bullet)$ and 1a- $d(\Delta)$ (monitored at the $\mathrm{F}_{1}$ band).

nents that were fitted to be 80 and $275 \mathrm{ps}$, respectively $\left(\chi^{2}=1.01\right)$. The rise time of the $\mathrm{F}_{2}$ band, within experimental error, is identical with the decay time of the $F_{1}$ band, further supporting the precursor-successor type of reaction mechanism. ESIPT dynamics were also obtained for $\mathbf{1 b}-\mathbf{d}$ at $298 \mathrm{~K}$ and the results are listed in Table 1.

The rate constants of $70-130 \mathrm{ps}^{-1}$ measured for $\mathbf{1 a}-\mathbf{d}$ are $\sim 2$ orders of magnitude smaller than that reported for typical ESIPT molecules in nonpolar solvents. ${ }^{4}$ Slower proton-transfer reaction may be in some way associated with its rather weak hydrogenbonding strength, and hence a long HB distance. Thus, one might initially suspect that this is simply due to a larger barrier along the reaction coordinate with only the proton motion involved. To test this possibility, the ESIPT rates in deuterated $(\mathrm{N}(1)-\mathrm{D})$ versions of $\mathbf{1 a}-\mathbf{d}$ were investigated. If only proton motion is involved in the reaction coordinate, a large deuterium isotope effect would be predicted. However, as shown in Figure 2, within experimental errors, our results reveal a negligible isotope effect.

Accordingly, more than proton motion must be involved in the reaction coordinate. Molecular modeling of $\mathbf{1 a}$ renders a relatively long $\mathrm{N}(1) \mathrm{H} \cdot \cdots \mathrm{N}\left(1^{\prime}\right)$ HB distance of $\sim 2.49 \AA$ with an $\mathrm{N}-\mathrm{H} \cdots \mathrm{N}$ angle of $\sim 93^{\circ} .11$ These results, in combination with the restricted orientation of the pyrazole $\mathrm{N}-\mathrm{H}$ bond, lead us to propose that the reaction coordinate does not couple directly with the $\mathrm{N}-\mathrm{H}$ stretching mode. Rather, it involves other skeletal motions such as in-plane bending modes, which change the relative position of atoms associated with the hydrogen bond, and hence channel into the proton-transfer process. In this case, the resulting effective tunneling mass should be greatly increased so that the deuteration of amino proton results in only a very small fractional increase in the tunneling mass. As a result, a negligible H/D isotope effect would be observed. We further carried out a series of temperaturedependent studies regarding reaction dynamics. As shown in Figure 2 , the reaction rate for $\mathbf{1 a}$ monitored by the decay dynamics of the $\mathrm{F}_{1}$ band or equivalently the rise dynamics of the $\mathrm{F}_{2}$ band revealed significant temperature dependence in the range of $298-203 \mathrm{~K}^{12}$ The logarithm plot for the ESIPT rate versus $1 / T$ is sufficiently linear, from which a nearly deuterium isotope independent barrier $(\Delta E \approx 2.03 \mathrm{kcal} / \mathrm{mol})$ and frequency factor $\left(\sim 3.8 \times 10^{11} \mathrm{~s}^{-1}\right)$ were deduced. Negligible N-D isotope ESIPT dynamics were also observed for $\mathbf{1 b}-\mathbf{d}$, and their corresponding $\Delta E$ are listed in Table 1. Upon increasing the electron-withdrawing ability in $\mathrm{R}_{\mathrm{b}}$ (see Scheme 1) the acidity of the N(1) $-\mathrm{H}$ proton should increase, and hence faster ESIPT dynamics is expected. In contrast, Table 1 shows a lack of correlation between $\Delta E$ and donating/accepting properties of $\mathrm{R}_{\mathrm{b}}$, indirectly supporting the skeletal reorganization facilitating ESIPT process. Further support was given by a similar Arrhenius plot for 1a in dry $\mathrm{CH}_{3} \mathrm{CN}$, and $\Delta E$ was deduced to be $\sim 2.90 \mathrm{kcal} /$ mol, consistent with that obtained in methylcyclohexane.

In conclusion, the results demonstrate a novel and unique system among ESIPT molecules where the intrinsic proton transfer is associated with a substantial energy barrier. The nature of the reaction potential surface in $\mathbf{1} \mathbf{a}-\mathbf{d}$ may be described by certain skeletal reorganization and hence is of a great theoretical challenge. This, in combination with the structural simplicity and diversity, makes the 5-(2-pyridyl)-1- $H$-pyrazole system an ideal model for probing ESIPT dynamics, which are believed to bring up a broad spectrum of interests in the proton-transfer field.

Supporting Information Available: Detailed experimental procedures, absorption, emission, time-resolved, and X-ray studies (PDF/ CIF). This material is available free of charge via the Internet at http:// pubs.acs.org.

\section{References}

(1) Weller, A. Z. Elektrochem. 1956, 60, 1144

(2) For recent reviews, see: (a) Scheiner, S. J. Phys. Chem. A 2000, 104 , 5898. (b) Waluk, J. Conformational aspects of intra- and intermolecular excited state proton transfer. In Conformational Analysis of Molecules in Excited States; Waluk, J., Ed.; Wiley-VCH: 2000. (c) Chou, P. T. J. Chin. Chem. Soc. 2001, 48, 651.

(3) The prohibition of ESIPT in the $n \pi^{*}$ state has been reported in several ESIPT molecules. For example, see ref $2 \mathrm{a}$

(4) For example, see: (a) Chudoba, C.; Riedle, E.; Pfeiffer, M.; Elsaesser, T. Chem. Phys. Lett. 1996, 263, 622. (b) Lochbrunner, S.; Wurzer, A. J.; Riedle, E. J. Chem. Phys. 2000, 112, 10699. (c) Chou, P. T.; Chen, Y. C.; Yu, W. S.; Chou, Y. H.; Wei, C. Y.; Cheng, Y. M. J. Phys. Chem. A 2001, 105, 1731. (d) Ameer-Beg, S.; Ormson, S. M.; Brown, R. G.; Matousek, P.; Towrie, M.; Nibbering, E. T. J.; Foggi, P.; Neuwahl, F. V. R. J. Phys. Chem. A 2001, 105, 3709. (e) Stock, K.; Bizjak, T.; Lochbrunner, S. Chem. Phys. Lett. 2002, 354, 409.

(5) One exceptional case is 3-hydroxyflavones possessing a five-memberedring $=\mathrm{O} \cdots \mathrm{H}-\mathrm{O}$ hydrogen bond. Ultrafast ESIPT was reported for 3-hydroxyflavone (3HF) in nonpolar solvents. ${ }^{4 \mathrm{~d}}$ Barrierless excited-state proton transfer in $3 \mathrm{HF}$ was also reported through the cyclic hydrogen bond with protic solvents such as methanol. ${ }^{6}$

(6) Schwartz, B. J.; Peteanu, L. A.; Harris, C. B. J. Phys. Chem. 1992, 96, 3591

(7) The ESIPT from phenol $\mathrm{O}-\mathrm{H}$ to a $\beta$-carbon atom is not included in this category, see: Lukeman, M.; Wan, P. J. Am. Chem. Soc. 2002, 124, 9458.

(8) For recent examples, see: (a) Kyrychenko, A.; Herbich, J.; Izydorzak, M.; Wu, F.; Thummel, R. P.; Waluk, J. J. Am. Chem. Soc. 1999, 121, 11179. (b) Herbich, J.; Kijak, M.; Zielińska, A.; Thummel, R. P.; Waluk, J. J. Phys. Chem. A 2002, 106, 2158.

(9) Taylor, C. A.; El-Bayoumi, A. M.; Kasha, M. Proc. Natl. Acad. Sci. U.S.A. 1969, 65, 253. Also, see refs $2 \mathrm{~b}$ and $2 \mathrm{c}$ for recent reviews.

(10) See Supporting Information for detailed syntheses and characterization.

(11) The calculation was based on the HF/6-31G(d', $\left.\mathrm{p}^{\prime}\right)$ level. X-ray data were not applied here due to the dimeric structure in a single crystal.

(12) Further decreasing temperatures resulted in microcrystals interferences. JA035382Y 